\title{
Fatores Relevantes para a Criação e Uso de Comunidades Virtuais de Aprendizagem: estudo em um projeto de pesquisa transnacional
}

\author{
Janice Kirner Providelo - PPGEU / UFSCAR, São Carlos SP - jkirner@gmail.com \\ Tereza Gonçalves Kirner - ICE / UNIFEI, Itajubá, MG - tgkirner@ gmail.com
}

Resumo. Atualmente, a educação vem sendo modificada, com vistas a adaptar-se às mudanças decorrentes da globalização. Universidades e centros de pesquisa, em busca de profissionais com habilidades específicas, vêm realizando projetos de âmbito internacional, que podem ser desenvolvidos com o suporte de comunidades virtuais de aprendizagem (CVAs). Apesar desses projetos estarem se disseminando, é necessário haver uma preocupação com requisitos específicos desses projetos, nos quais os participantes estão geograficamente distribuídos. Este artigo busca elucidar os fatores que podem interferir no sucesso de CVEs relacionadas com projetos transacionais. Um estudo empírico é apresentado, os resultados são discutidos e diretrizes para auxiliar a criação e uso de VLCs são propostas.

Palavras-chaves: comunidades virtuais de aprendizagem; requisitos de comunidades virtuais de aprendizagem; projetos transnacionais.

\section{Relevant Issues to the Creation and Use of Learning Virtual Communities: a study in a transnational research project}

\begin{abstract}
Nowadays, education has been being modified to adapt to the changes caused by globalization. Universities and research centers, searching for professionals with specific abilities, have been carrying international projects, which can be developed with the support of Virtual Learning Communities (VLC). Although this type of project is disseminating, it is necessary to be concerned with specific requirements and possible obstacles that can occur in such projects, in which the participants are geographically distributed. Therefore, this paper aims at contributing to elucidate the factors that interfere in the success of VLCs related to transnational projects. An empirical study is presented, the results are discussed and some guidelines to help the creation and utilization of VLCs are proposed.
\end{abstract}

Keywords: virtual learning communities; requirements of virtual learning communities; transnational projects.

\section{Introdução}

Atualmente, as universidades e centros de pesquisa têm buscado ajustar-se e desenvolver estratégias que atendam rapidamente às mudanças tecnológicas e à demanda por profissionais com habilidades específicas. Assim, têm surgido projetos de pesquisa de âmbito transnacional, desenvolvidos com o suporte de Comunidades Virtuais de Aprendizagem (CVAs). As CVAs representam uma alternativa aos modelos 
tradicionais de ensino-aprendizagem, sendo definidas como grupos descentralizados de pessoas que se auto-organizam, com o objetivo principal de apoiar-se mutuamente para a realização eficaz de atividades construtivas de aprendizagem. (Afonso, 2001).

Este trabalho teve como objeto de estudo o projeto de pesquisa transnacional Cycling Academic Network (CAN), que envolve um convênio colaborativo que faz parte do Bicycle Partnership Program, criado pela organização holandesa Interface for Cycling Expertise (I-CE) para promover pesquisas sobre o planejamento e o transporte cicloviário, com foco em países em desenvolvimento. Trata-se de um projeto gerenciado por universidades e centros de pesquisas da Holanda, que envolve participantes da Índia, África do Sul e Brasil (I-CE, 2008). O CAN teve início em 2009, com dois estudantes de doutorado de cada país participante, que trabalham na Holanda colaborativamente por um período coincidente de três a seis meses a cada ano, depois retornam aos seus países de origem. Durante o período em que permanecem em seus países, os trabalhos continuam a ser desenvolvidos pelos participantes. Sendo assim, é essencial que haja o apoio de uma Comunidade Virtual de Aprendizagem que funcione eficientemente.

A presente pesquisa procurou responder a seguinte pergunta: "Quais os fatores que interferem na criação e uso bem sucedido de uma Comunidade Virtual de Aprendizagem desenvolvida para apoiar projetos de pesquisa transnacionais?" A pesquisa foi realizada e os resultados obtidos permitiram propor uma lista de diretrizes, que pode ser útil para desenvolvedores e usuários de CVAs voltadas para projetos de pesquisa similares aos enfocados.

A seção 2 deste trabalho contém os aspectos principais da fundamentação teórica da pesquisa, a seção 3 resume a metodologia utilizada no estudo, a seção 4 aborda a análise e discussão dos resultados e, por fim, a seção 5 apresenta as considerações finais.

\section{Fundamentos}

De acordo com Afonso (2001) as comunidades de aprendizagem apresentam-se como uma alternativa aos modelos tradicionais de ensino-aprendizagem, constituindo-se de "grupos descentralizados de sujeitos que se auto-organizam em comunidades funcionais e estáveis, e que cuja meta principal é o apoio mútuo para o desenvolvimento eficaz de actividades construtivas de aprendizagem".

A construção de comunidades de aprendizagem é um problema que envolve pessoas, e, consequientemente, objetivos, idéias, emoções, disponibilidades, confiança, relacionamentos e interatividade (Andrade, 2001). Assim, pode-se dizer que o sucesso da criação das comunidades virtuais de aprendizagem está ligado a princípios básicos da colaboração humana. Neste sentido, o uso de tecnologias de informação e comunicação é fundamental para viabilizar a interação e a colaboração entre os participantes.

Comunidades virtuais de aprendizagem são grupos que interagem com o intuito de atingir quatro objetivos: informar-se, organizar comunicações, obter e aplicar conhecimento e realizar tarefas de transformação com propósitos específicos. Essas comunidades envolvem participantes e recursos visando oferecer serviços, organizar projetos, conduzir processos e disponibilizar recursos (Weber, 2006).

As comunidades virtuais de aprendizagem representam uma estratégia promissora para o compartilhamento de experiências, a geração de aprendizagem e a produção de conhecimento em projetos transnacionais (Bohme, 2005; Na Ubon, 2002). Um projeto transnacional é uma estrutura, criada temporariamente, composta de 
indivíduos de diferentes nacionalidades e experiências culturais, que atuam de forma colaborativa, a fim de atingir objetivos comuns. Esse projeto tem o propósito de gerar conhecimento especializado, produtos ou serviços, que serão direcionados para os diferentes países envolvidos ou para o mercado mundial (Adenfelt, 2006).

Os principais obstáculos que os projetos transnacionais enfrentam, de acordo com Adenfelt (2006) são: distância física dos participantes; fusos horários diferentes; diferenças culturais; uso de idiomas diferentes; estruturas tecnológicas diferentes; falta de recursos de apoio para a geração do conhecimento; falta de recursos para facilitar o trabalho colaborativo. Apesar da diversidade, peculiar a esses projetos, há necessidade de produção de resultados conjuntos e de objetivos comuns (Bohme, 2005).

\section{Desenvolvimento da pesquisa}

\subsection{Tipo de pesquisa e participantes}

A presente pesquisa pode ser definida como pesquisa exploratória (quanto aos fins) e pesquisa de campo (quanto aos meios). Adicionalmente, pode também ser classificada, quanto à técnica, como uma pesquisa empírica qualitativa (Vergara, 2006).

O ambiente da pesquisa de campo utilizado foi o grupo de alunos de doutorado do projeto $\mathrm{CAN}$, descrito anteriormente. O projeto inclui a participação de alunos de doutorado de três países-alvo (Brasil, Índia e África do Sul). Como o projeto teve início em 2009, conta somente com a primeira turma de seis alunos, que será expandida futuramente. Outros participantes do projeto CAN incluem orientadores locais, orientadores gerais e colaboradores.

Os alunos de doutorado foram escolhidos como sujeitos da pesquisa, uma vez que serão direta e continuamente afetados pela criação e uso da CVA pretendida. A amostra de pesquisa foi definida pelo critério de acessibilidade indicado por Vergara (2006), no qual os elementos da amostra são selecionados pela facilidade de acesso a eles, sem nenhum procedimento estatístico. Assim, os respondentes foram cinco alunos que participam atualmente do projeto CAN, ou seja, a primeira turma de alunos de doutorado do projeto, com exceção da autora principal da presente pesquisa.

\subsection{Coleta de Dados}

Para a coleta de dados, foram utilizadas entrevistas individuais, apoiadas por um roteiro de questões, apresentado a seguir:

- Quais facilidades ou vantagens você percebe no desenvolvimento do projeto CAN por meio de uma Comunidade Virtual de Aprendizagem?

- Quais dificuldades ou desvantagens você percebe no desenvolvimento do projeto CAN por meio de uma Comunidade Virtual de Aprendizagem?

- O que uma Comunidade Virtual de Aprendizagem deveria incluir para facilitar a interação entre os participantes do projeto CAN? E o que poderia dificultar essa interação?

- O que poderia facilitar a colaboração entre os participantes do projeto CAN na aprendizagem e construção coletiva do conhecimento (incluindo a geração de idéias, o desenvolvimento do projeto e a disseminação dos resultados)?

- O que poderia dificultar tal colaboração e a aprendizagem e construção coletiva de conhecimento? 
Devido à distância em que se encontravam os respondentes, as entrevistas foram iniciadas pessoalmente em um evento em que todos se encontraram e finalizadas por $e$ mail e chat. Antes do início de cada entrevista, o respondente foi informado quanto ao objetivo e a relevância da pesquisa e a garantia de anonimato dos participantes. A língua utilizada para as entrevistas foi o inglês.

\subsection{Análise dos dados}

A análise dos dados foi realizada por meio da técnica de "análise de conteúdo categorial" das entrevistas, a partir de categorias comuns às respostas dos sujeitos. Este tipo de análise leva em consideração a totalidade de um 'texto', passando-o pelo crivo da classificação, segundo a freqüência de presença (ou de ausência) de itens de sentido (Bardin, 1995).

As categorias temáticas principais e respectivas categorias secundárias, definidas para analisar as respostas referentes às expectativas e necessidades dos participantes do projeto CAN quanto às facilidades e dificuldades relativas ao desenvolvimento do projeto com o suporte da CVA pretendida, são apresentadas a seguir.

\section{a) Categoria 1 - Vantagens e facilidades relativas ao desenvolvimento do projeto com o suporte da CVA}

- Compartilhamento de conhecimento: contribuição dos participantes (oriundos de diferentes países) para a construção do conhecimento;

- Agilidade na construção do conhecimento: com base na contribuição individual de vários participantes;

- Construção de uma base de dados de apoio: base de dados para facilitar o uso compartilhado de referências e textos;

- Troca de informações com o meio externo: compartilhamento de informações sobre eventos, projetos, etc. e disseminação de resultados do projeto;

- Redução dos custos do projeto: através da diminuição de viagens para reuniões presenciais.

b) Categoria 2 - Desvantagens e dificuldades relativas ao desenvolvimento do projeto com o suporte da CVA

- Comunicação assíncrona: dificuldades devido à lentidão ocasionada por restrições da tecnologia da informação e/ou comunicação pessoal;

- Comunicação síncrona: dificuldades devido à diferença de fusos horários;

- Manutenção da CVA: necessidade de pessoal e outros recursos para administrar e manter a CVA;

- Interação não presencial: dificuldade de motivação e envolvimento dos participantes;

- Diferenças de responsabilidades e calendários: dificuldade de ajustar as atividades pessoais dos participantes e respeitar os calendários de ano letivo e feriados dos diferentes países.

c) Categoria 3 - Ferramentas de TIC para interação e colaboração na CVA 
- Fóruns de discussão: ferramentas de comunicação assíncrona, com ou sem moderação, para discussão de aspectos gerais e específicos;

- Chat e videoconferência: ferramentas de comunicação síncrona, que possibilitam a comunicação em tempo real entre grupos de pessoas, independente de suas localizações geográficas, em texto, áudio ou em áudio e vídeo simultaneamente;

- Softwares de apoio: softwares para o uso compartilhado de material bibliográfico e de análise de dados e para a organização e acompanhamento do projeto.

\section{d) Categoria 4 - Aspectos pessoais e culturais da interação e colaboração na CVA}

- Afetividade: relação afetiva entre os participantes da comunidade virtual de aprendizagem;

- Diferenças individuais de interesse e comportamento: podem resultar em prevalecimento de uma visão específica;

- Competitividade entre os participantes: pode comprometer o andamento do projeto, gerar ocultação de informação, etc.

- Diferenças culturais: provenientes de religião, costumes e outros, dos diferentes países;

- Linguagem: necessidade do domínio de uma língua comum entre os participantes.

\section{e) Categoria 5 - Administração da CVA}

- Estabelecimento de regras: contanto que sejam conhecidas e aceitas por todos os participantes;

- Participação de especialistas: para contribuir em sessões específicas;

- Estabelecimento de liderança: visando a manutenção de um foco comum;

- Acesso à Internet: necessidade de viabilizar o acesso fácil e contínuo à Internet, considerando-se também o custo e a qualidade do serviço.

Após a etapa de exploração do material, foi realizada a última etapa de análise de conteúdo indicada por Bardin (1995), que incluiu o tratamento dos resultados, inferência e interpretação.

\section{Discussão dos resultados}

Os resultados da análise de conteúdo dos dados obtidos nas entrevistas foram esquematizados em quadros, apresentados a seguir, de acordo com cada categoria temática de análise. Os quadros são compostos pelos temas principais e secundários de cada categoria, as respectivas frequiências de explicação e exemplos de verbalizações.

\subsection{Categoria 1}

O Quadro 1 sintetiza os resultados obtidos referentes à Categoria 1, compreendendo as vantagens e facilidades consideradas pelos participantes.

Nesta categoria, houve cinco manifestações sobre o compartilhamento de conhecimento como vantagem de uma possível comunidade virtual de aprendizagem para apoiar o projeto CAN. Na opinião dos entrevistados, esse compartilhamento, viabilizado pela CVA, traria um enriquecimento do conhecimento gerado, com base na troca de experiências advindas de diferentes contextos. 
De acordo com os entrevistados, uma CVA poderia reduzir os custos do projeto, além de possibilitar a troca de informações importantes com o meio externo, tais como notícias sobre conferências, bolsas, atividades, projetos, publicações, carreiras, etc. Cada uma destas categorias secundárias obteve duas manifestações dos participantes.

\section{Quadro 1. Vantagens e facilidades relativas ao projeto por meio da CVA}

\begin{tabular}{|l|c|l|}
\hline \multicolumn{1}{|c|}{ Temas } & Qtde & \multicolumn{1}{c|}{ Exemplos de verbalizações } \\
\hline $\begin{array}{l}\text { 1. Compartilhamento de } \\
\text { conhecimento }\end{array}$ & 5 & $\begin{array}{l}\text { "O projeto CAN se beneficiaria e seus resultados seriam enriquecidos } \\
\text { através da experiência e conhecimento dos seus indivíduos, manifestada } \\
\text { na CVA." }\end{array}$ \\
\hline $\begin{array}{l}\text { 2. Agilidade na construção } \\
\text { do conhecimento }\end{array}$ & 1 & "... a CVA reduz o tempo para alcançar o conhecimento pretendido." \\
\hline $\begin{array}{l}\text { 3. Construção de uma base } \\
\text { de dados de apoio }\end{array}$ & 1 & $\begin{array}{l}\text { "Seria de grande valor a disponibilização de uma base de dados sobre } \\
\text { tópicos relacionados (ciclismo) incluindo referências, artigos e relatórios, } \\
\text { para uso comum." }\end{array}$ \\
\hline $\begin{array}{l}\text { 4. Troca de informações } \\
\text { com o meio externo }\end{array}$ & 2 & $\begin{array}{l}\text { "... troca de informações sobre conferências, bolsas, atividades, projetos, } \\
\text { publicações, carreiras, etc. podem levar à abertura da comunidade." }\end{array}$ \\
\hline $\begin{array}{l}\text { 5. Redução dos custos do } \\
\text { projeto }\end{array}$ & 2 & $\begin{array}{l}\text { "Ao invés de organizar workshops ou sessões conjuntas, que custam } \\
\text { caro, é mais fácil, através de uma CVA, prover uma plataforma para } \\
\text { interação entre os pesquisadores." }\end{array}$ \\
\hline
\end{tabular}

\subsection{Categoria 2}

$\mathrm{O}$ Quadro 2 resume os resultados obtidos referentes à Categoria 2, enfocando desvantagens e dificuldades vislumbradas pelos participantes.

$\mathrm{Na}$ categoria 2, o maior número de manifestações dos entrevistados refere-se às desvantagens e dificuldades representadas pela comunicação síncrona em uma possível comunidade virtual de aprendizagem para apoiar o projeto CAN. Três participantes destacaram problemas provenientes de diferentes fusos horários e rotinas de trabalho.

Os entrevistados indicaram também possíveis problemas relacionados com a comunicação assíncrona e interação não presencial. Cada uma destas categorias secundárias obteve duas manifestações dos participantes. As manifestações ressaltam que pode haver atrasos nas respostas dos e-mails, além de dificuldades para se manter discussões produtivas devido à barreira da distância entre os participantes da CVA.

Outras desvantagens indicadas pelos entrevistados envolvem a criação e manutenção da CVA e diferenças de responsabilidades e calendários individuais dos participantes. Cada uma destas categorias secundárias obteve uma manifestação dos entrevistados. Foi destacada a necessidade de uma pessoa responsável pela CVA, e também que cada país possui os seus próprios calendários, além das diferentes prioridades de cada participante.

\section{Quadro 2. Desvantagens e dificuldades relativas ao projeto por meio da CVA}

\begin{tabular}{|l|c|l|}
\hline \multicolumn{1}{|c|}{ Temas } & Qtde & \multicolumn{1}{c|}{ Exemplos de verbalizações } \\
\hline 1. Comunicação assíncrona & 2 & "O processo de troca de $e$-mails é lento na rede..." \\
\hline 2. Comunicação síncrona & 3 & $\begin{array}{l}\text { "... realização de chats é difícil devido às diferenças de tempos } \\
\text { convenientes para cada um ou às diferenças de fuso horário." }\end{array}$ \\
\hline
\end{tabular}

V. $8 \mathrm{~N}^{\mathrm{o}} 3$, dezembro, 2010 


\begin{tabular}{|l|c|l|}
\hline 3. Manutenção da CVA & 1 & $\begin{array}{l}\text { "Uma dificuldade repousa no fato de que alguém tem que organizar a } \\
\text { comunidade e dedicar tempo e esforço para a sua manutenção." }\end{array}$ \\
\hline 4. Interação não presencial & 2 & $\begin{array}{l}\text { "... pode ser difícil manter um ambiente de aprendizagem vibrante sem o } \\
\text { contato real entre os membros do grupo. A comunicação pode ficar mais } \\
\text { difícil quando os participantes não estão juntos." }\end{array}$ \\
\hline $\begin{array}{l}\text { 5. Diferenças de responsabi- } \\
\text { lidades e calendários }\end{array}$ & 1 & $\begin{array}{l}\text { "Pode haver dificuldade no andamento do projeto devido à diferentes } \\
\text { prioridades e prazos dos participantes e das diferenças de contexto } \\
\text { (calendário acadêmico, feriados) de cada país." }\end{array}$ \\
\hline
\end{tabular}

\subsection{Categoria 3}

O Quadro 3 apresenta os resultados obtidos referentes à categoria 3, enfocando as ferramentas que seriam úteis na CVA, segundo a percepção dos respondentes.

Nesta categoria, foram destacados três tipos de ferramentas de TIC desejáveis para interação e colaboração na comunidade virtual de aprendizagem: fóruns de discussão, chat e videoconferência e softwares de apoio.

As ferramentas de chat e videoconferência receberam quatro verbalizações, destacando que elas poderiam contribuir para a aprendizagem e para manter $o$ envolvimento e interesse dos participantes nas atividades. Os fóruns poderiam facilitar a discussão dos assuntos de maneira organizada, podendo também servir para tratamento de assuntos específicos, como, por exemplo, questões de ordem administrativa. Dependendo da situação, poderiam ser viabilizados fóruns abertos ou fechados, com ou sem moderação. Três participantes manifestaram-se sobre esta categoria secundária.

Três participantes manifestaram-se a favor da inclusão de ferramentas de software para apoiar as atividades de obtenção, compartilhamento e organização de literatura, além de softwares para auxiliar a análise de dados das pesquisas.

\section{Quadro 3. Ferramentas de TIC para interação e colaboração na CVA}

\begin{tabular}{|l|c|l|}
\hline \multicolumn{1}{|c|}{ Temas } & Qtde & \multicolumn{1}{|c|}{ Exemplos de verbalizações } \\
\hline 1. Fóruns de discussão & 3 & $\begin{array}{l}\text { "Para assegurar o funcionamento do grupo, uma plataforma de comunicação } \\
\text { comum com fóruns de discussão online devem ser estabelecidos." }\end{array}$ \\
\hline $\begin{array}{l}\text { 2. Chat e videoconfe- } \\
\text { rência }\end{array}$ & 4 & $\begin{array}{l}\text { "Videoconferências - onde as pessoas possam expressar suas visões, } \\
\text { esclarecer idéias e manter os participantes envolvidos e interessados." }\end{array}$ \\
\hline 3. Softwares de apoio & 3 & $\begin{array}{l}\text { "Programas comuns para compartilhamento de dados e administração de } \\
\text { atividades - auxílio e assistência para compartilhamento e organização da } \\
\text { literatura e para análise de dados." }\end{array}$ \\
\hline
\end{tabular}

\subsection{Categoria 4}

O Quadro 4 sintetiza os resultados obtidos para a categoria 4, compreendendo a percepção dos respondentes sobre aspectos pessoais e culturais que podem afetar a utilização da CVA.

Nesta categoria, três entrevistados manifestaram-se a respeito das diferenças individuais, de interesse e de comportamento dos participantes do projeto, que necessitam ser bem administrados no contexto da CVA. Estas diferenças individuais poderiam prejudicar o compartilhamento do conhecimento.

Dois entrevistados indicaram também aspectos de afetividade e de diferenças culturais como possíveis geradores de dificuldades no uso da CVA para apoiar o projeto. Foi destacado que é importante que os membros tenham a oportunidade de se conhecer pessoalmente visando uma interação e comunicação mais efetiva. Além disso, 
deve-se gerenciar adequadamente as diferenças culturais dos membros originários de diferentes países e continentes.

Os aspectos de linguagem e de comportamento competitivo foram citados, cada um por um participante. A competitividade poderia, na visão de um dos entrevistados, prejudicar o compartilhamento de informações. A linguagem, por sua vez, pode ser um problema, se todos os participantes do projeto não dominarem uma língua comum.

\section{Quadro 4. Aspectos pessoais e culturais da interação e colaboração na CVA}

\begin{tabular}{|l|c|l|c|}
\hline \multicolumn{1}{|c|}{ Temas } & Qtde & \multicolumn{1}{|c|}{ Exemplos de verbalizações } \\
\hline 1. Afetividade & 2 & $\begin{array}{l}\text { "...(os membros do grupo) devem, de preferência, ter se conhecido } \\
\text { pessoalmente. (...) É mais fácil colaborar com pessoas que você conhece } \\
\text { e gosta." }\end{array}$ \\
\hline $\begin{array}{l}\text { 2. Diferenças individuais de } \\
\text { interesse e comportamento }\end{array}$ & 3 & $\begin{array}{l}\text { "Indivíduos com interesses variados e diferentes estilos de } \\
\text { comportamento podem ter problema em relacionar-se com os outros, } \\
\text { prejudicando o compartilhamento do conhecimento." }\end{array}$ \\
\hline $\begin{array}{l}\text { 3. Competitividade entre os } \\
\text { participantes }\end{array}$ & 1 & $\begin{array}{l}\text { "O sentimento de competitividade faz surgir problemas no } \\
\text { compartilhamento de informação." }\end{array}$ \\
\hline 4. Diferenças culturais & 2 & $\begin{array}{l}\text { "Diferenças culturais também podem ser um problema e devem ser bem } \\
\text { gerenciadas." }\end{array}$ \\
\hline 5. Linguagem & 1 & $\begin{array}{l}\text { "A língua pode ser um problema - todos os membros devem estar aptos a } \\
\text { se comunicar facilmente através de uma língua comum." }\end{array}$ \\
\hline
\end{tabular}

\subsection{Categoria 5}

O Quadro 5 mostra os resultados obtidos referentes à categoria 5, enfocando os aspectos relativos ao funcionamento da CVA, segundo a percepção dos respondentes.

Nesta categoria, os participantes destacaram a importância de haver regras vigentes no contexto da CVA, desde que elas sejam aceitas por todos os membros do projeto, sem imposições. Dois entrevistados manifestaram-se a esse respeito. Além disso, o estabelecimento de liderança e a viabilidade de acesso à Internet também receberam duas verbalizações cada um. Algum tipo de liderança foi apontada como forma de manter um foco comum nas discussões e demais atividades do projeto. $\mathrm{O}$ acesso à Internet, com qualidade de comunicação e custos compatíveis com o orçamento do projeto, foi indicado como aspecto administrativo a ser considerado para a implantação de uma CVA.

Um dos entrevistados destacou que a participação de especialistas convidados na CVA poderia contribuir para a construção do conhecimento em assuntos específicos, o que poderia ser obtido através da organização de sessões de discussão, conferências, etc.

\section{Quadro 5. Administração da CVA}

\begin{tabular}{|l|c|l|}
\hline \multicolumn{1}{|c|}{ Temas } & Qtde & \multicolumn{1}{c|}{ Exemplos de verbalizações } \\
\hline $\begin{array}{l}\text { 1. Definição de regras aceitas } \\
\text { por todos os participantes }\end{array}$ & 2 & $\begin{array}{l}\text { "Um protocolo ou qualquer tipo de regras impostas aos participantes } \\
\text { pode complicar a participação." }\end{array}$ \\
\hline $\begin{array}{l}\text { 2. Participação de } \\
\text { especialistas }\end{array}$ & 1 & $\begin{array}{l}\text { "Organizar sessões conjuntas com ajuda de especialistas pode melhorar } \\
\text { a colaboração." }\end{array}$ \\
\hline 3. Estabelecimento de & 2 & "Um fórum com discussões ativas e com moderação pode ajudar a \\
\hline
\end{tabular}

V. $8 \mathrm{~N}^{\mathrm{o}}$ 3, dezembro, 2010 


\begin{tabular}{|l|c|l|}
\hline liderança & & $\begin{array}{l}\text { delinear o projeto em várias dimensões, além de manter um foco } \\
\text { comum." }\end{array}$ \\
\hline $\begin{array}{l}\text { 4. Viabilidade de acesso à } \\
\text { Internet }\end{array}$ & 2 & $\begin{array}{l}\text { "O acesso à Internet não é universal e pode se tornar caro para alguns } \\
\text { participantes. Como resultado, alguns membros podem contribuir com } \\
\text { o projeto com menos facilidade do que outros." }\end{array}$ \\
\hline
\end{tabular}

Ao final da análise dos dados, os resultados obtidos foram sintetizados, produzindo-se uma lista de diretrizes que pode ser útil para a criação e uso de Comunidades Virtuais de Aprendizagem focadas em projetos de pesquisa transnacionais.

\section{Considerações finais}

Os resultados da pesquisa permitiram a definição de diretrizes para apoiar a criação de Comunidades Virtuais de Aprendizagem focadas no apoio a projetos de pesquisa transnacionais. A lista de diretrizes é apresentada a seguir.

i. Disponibilizar recursos de TIC, incluindo: fórum, para comunicação assíncrona entre os participantes; chat e videoconferência com apoio de imagem e voz, para comunicação síncrona; e software, para apoiar a aquisição, armazenamento e disseminação do conhecimento.

ii. Levar em consideração as diferenças de fuso horário, diferenças de ano letivo e calendários de feriados para a definição de reuniões, prazos e atividades do projeto.

iii. Levar em consideração aspectos de qualidade dos serviços de Internet e custos destes serviços para os participantes do projeto, com indicativos sobre o custeio destas despesas.

iv. Levar em consideração a necessidade de criação e manutenção da CVA, incluindo recursos financeiros e de pessoal para isso.

v. Considerar a participação de especialistas externos ao projeto, que poderiam ser convidados para reuniões por meio de videoconferências, chats e fóruns.

vi. Promover reuniões e discussões via chat e fórum, com a mediação de membros do projeto ou convidados externos, sempre que se fizer necessário, como forma de agilizar e enriquecer o processo de geração e compartilhamento do conhecimento.

vii. Promover, sempre que possível, uma reunião presencial, no início do projeto, para que os membros se conheçam pessoalmente. Isso ajudará no entrosamento entre as pessoas, o que contribuirá para a criação de um ambiente de confiança, destacado como importante para a interação e colaboração por meio da CVA.

viii. Considerar a indicação de um membro, que poderia ser escolhido entre os membros do grupo, que ficaria responsável por promover a interação e colaboração dentro da 
CVA. Esse membro deveria atuar na solução de aspectos decorrentes das diferenças individuais dos participantes.

ix. Estabelecer uma língua oficial para todo o tipo de participação na CVA e produção de materiais e resultados.

x. Promover, no contexto da CVA, por meio de regras formais e medidas informais, a aceitação das diferenças individuais inerentes aos participantes de um projeto transnacional.

Espera-se, assim, que este estudo possa contribuir para a criação uso de CVAs bem sucedidas, dirigidas para projetos transnacionais com participantes geograficamente distribuídos, não somente no caso do projeto CAN, mas também para outros projetos similares. Além disso, espera-se agregar informações importantes ao tema em questão, que tem sido pouco abordado em pesquisas publicadas internacionalmente.

\section{Referências bibliográficas}

ADENFELT, M.; LAGERSTROM, K. (2006) Enabling knowledge creation and sharing in transnational projects. International Journal of Project Management, Amsterdam: Elsevier, v. 24, p. 191-198, 2006.

AFONSO, A. (2001) Comunidades de aprendizagem: um modelo para a gestão da aprendizagem. In: CONFERÊNCIA INTERNACIONAL DE TECNOLOGIAS DE INFORMAÇÃO E COMUNICAÇÃO NA EDUCAÇÃO, II, 2001, Braga, PT. Anais. p. 427-432.

ANDRADE, A.; MACHADO, A.B. Comunidades de aprendizagem. In: CONFERÊNCIA INTERNACIONAL DE TECNOLOGIAS DE INFORMAÇÃO NA EDUCAÇÃO, II, 2001, Braga, PT. Anais. p. 451-461.

BARDIN, L. Análise de conteúdo. Lisboa: Editora 70. 1995.

BOHME, K. The ability to learn in transnational projects. Informationen zur Raumentwicklung, Berlim. 2005.

I-CE. Interface for Cycling Expertise. Disponível em: <www.cycling.nl>, Acesso em: 28 mar. 2010.

NA UBON, A.; KIMBLE, C. (2002) Knowledge management in online distance education. In: CONFERENCE ON E-LEARNING IN NETWORKED LEARNING, 2002, Sheffield, UK. Proceedings. p. 465-473.

VERGARA, S.C. Projetos e Relatórios de Pesquisa. 7 ed., São Paulo: Atlas. 2006. 
Weber, J.B., Vásquez, J. and Romero, R. Application of a knowledge management network in distance education. In: INTERNATIONAL CONFERENCE ON MULTIMIDIA AND INFORMATION TECHNOLOGIES IN EDUCATION, IV, 2006, Sevilha, ES. Proceedings. p. 11-16. 